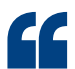

an alternative
stem cell
population
in the small
intestine can
compensate
for the loss
of the fast-
cycling LGR5-
expressing
CBCs

\title{
The ‘backup' intestinal stem cell
}

Two stem cell populations have been identified in the small intestine epithelium: fast-cycling stem cells termed crypt base columnar cells (CBCs), which express Leu-rich repeat-containing GPCR 5 (LGR5) and are ubiquitous; and slowercycling stem cells, which express the polycomb protein BMI1 and are rarer. de Sauvage, Klein and colleagues investigated the functions and relationship of the two populations and found that BMI1-expressing cells can repopulate the epithelium of the small intestine in the absence of LGR5-expressing CBCs.

To investigate the function of CBCs, the authors used mice in which the diphtheria toxin receptor (DTR) was knocked into the Lgr5 locus to allow the depletion of CBCs expressing LGR5 following administration of diphtheria toxin

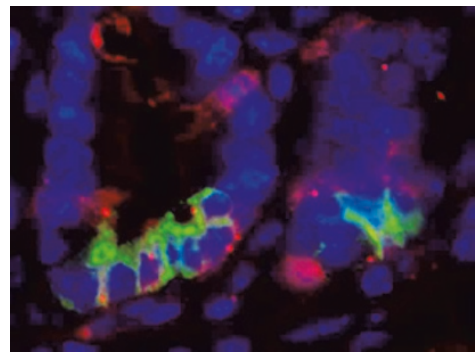

BMI1-expressing cells (tagged with $\beta$-galactosidase; red) can give rise to all cell types in the intestinal crypt, including LGR5-expressing cells (green). Image courtesy of $\mathrm{H}$. Tian, Genentech, San Francisco, California, USA.
(LGR5 $5^{\mathrm{DTR} /+}$ mice). As expected, LGR5-expressing cells were ablated 24 hours after injection of diphtheria toxin, and this effect was maintained after 10 days of exposure. Surprisingly, however, the architecture of the intestinal crypt was similar in control mice and in transgenic mice exposed to the toxin for 10 days. This normal intestinal crypt architecture was not due to the action of residual CBCs that had escaped ablation, indicating that another cell type may have compensated for the loss of CBCs.

So, could BMI1-expressing cells be responsible for maintaining a normal intestinal crypt architecture after CBC ablation? To answer this question, the authors modified the transgenic mice further to allow the tracking of BMI1 expression with green fluorescent protein (GFP). They observed that, following depletion of LGR5-expressing CBCs, the total number of BMI1-expressing cells increased threefold, and their numbers per intestinal crypt increased by $40 \%$ compared with control mice. Importantly, the numbers of crypts that were fully labelled with GFP in the first $5 \mathrm{~cm}$ of the duodenum increased 15-fold upon diphtheria toxin administration. This indicates that BMI-expressing cells can give rise to all cell types in the intestinal crypt in the absence of LGR5-expressing
CBCs, at least in this part of the intestine, and that production of progeny under these experimental conditions does not involve passing through an LGR5-positive intermediate.

Finally, the authors examined whether BMI1-expressing cells can also give rise to LGR5-expressing cells under normal conditions using LGR5 $5^{\mathrm{DTR} /+}$ mice that could be induced by tamoxifen to express BMI1 tagged to $\beta$-galactosidase and LGR5 tagged to GFP. They were able to identify cells expressing both BMI1 and LGR5 24 hours after administration of tamoxifen, and the number of these doubled after 48 hours. Moreover, when the mice were treated with diphtheria toxin for 6 days and allowed to recover for 72 hours, the authors observed that the BMI1-expressing cells were able to give rise to new LGR5-expressing cells.

Together, these findings reveal that an alternative stem cell population in the small intestine can compensate for the loss of the fast-cycling LGR5-expressing CBCs in normal and pathological conditions, leading to the generation of a complete intestinal epithelium.

Rachel David

ORIGINAL RESEARCH PAPER Tian, H. et al. A reserve stem cell population in small intestine renders Lgr5-positive cells dispensable. Nature 18 Sep 2011 (doi:10.1038/nature10408) 